\title{
Anharmonic phonon decay in cubic GaN
}

\author{
R. Cuscó and N. Domènech-Amador \\ Institut Jaume Almera (ICTJA-CSIC), Consejo Superior de Investigaciones Científicas, 08028 Barcelona, Spain
}

S. Novikov and C. T. Foxon

School of Physics and Astronomy, University of Nottingham, Nottingham NG7 2RD, United Kingdom

L. Artús

Institut Jaume Almera (ICTJA-CSIC), Consejo Superior de Investigaciones Científicas, 08028 Barcelona, Spain

(Received 26 May 2015; revised manuscript received 10 July 2015; published 21 August 2015)

\begin{abstract}
We present a Raman-scattering study of optical phonons in zinc-blende (cubic) GaN for temperatures ranging from 80 to $750 \mathrm{~K}$. The experiments were performed on high-quality, cubic $\mathrm{GaN}$ films grown by molecular-beam epitaxy on GaAs (001) substrates. The observed temperature dependence of the optical phonon frequencies and linewidths is analyzed in the framework of anharmonic decay theory, and possible decay channels are discussed in the light of density-functional-theory calculations. The longitudinal-optical (LO) mode relaxation is found to occur via asymmetric decay into acoustic phonons, with an appreciable contribution of higher-order processes. The transverse-optical mode linewidth shows a weak temperature dependence and its frequency downshift is primarily determined by the lattice thermal expansion. The LO phonon lifetime is derived from the observed Raman linewidth and an excellent agreement with previous theoretical predictions is found.
\end{abstract}

DOI: 10.1103/PhysRevB.92.075206

PACS number(s): 78.30.Fs, 63.20.-e

\section{INTRODUCTION}

The group-III nitride semiconductors with the zinc-blende structure are attracting much interest as promising candidates to overcome the built-in piezoelectric fields and spontaneous polarization effects that limit the efficiency of light-emitting devices based on wurtzite heterostructures with (0001) orientation [1,2]. Because of their symmetry, cubic group-III nitride heterostructures grown in a (001) direction are not affected by the quantum confined Stark effect. The absence of built-in fields is expected to yield a superior stability of the emission wavelength with increasing current and to increase the radiative efficiency in the well. The zinc-blende polytype of $\mathrm{GaN}$, with a band gap $0.2 \mathrm{eV}$ lower than that of the wurtzite phase [3] and higher hole mobility [4], is being considered as an alternative to the growth along nonpolar axes of wurtzite to address the droop problem in green light-emitting diodes [2]. The lower band gap of the cubic form requires less In alloying to reach the green emission region and, because of the absence of polarization fields, wider wells can be used where the carrier density is lower and hence the Auger nonradiative recombination rate [5] is reduced.

Recently, the optical properties of cubic GaN (c-GaN) have been thoroughly investigated in the near-band-gap excitonic region by means of temperature-dependent photoluminescence, photoreflectance, and ellipsometry [3]. These studies probe the electronic band structure of the material and provide valuable data for the design of devices based on c-GaN. However, the temperature dependence of the phonons in c-GaN has not received much attention. Studies of the temperature dependence of phonons yield relevant information about the electron-phonon interaction, which determines the energy-loss rate from electrons to the lattice via optical-phonon emission and is a key parameter in the design of high-speed devices. At high current densities, the energy loss of hot electrons to the lattice gives rise to self-heating effects which are deleterious to device performance. In polar lattices, hot-carrier relaxation takes place primarily by emission of zone-center longitudinaloptical (LO) phonons via Fröhlich interaction [6]. Depending on the LO phonon lifetimes, a nonequilibrium hot-phonon population may build up, leading to phonon reabsorption and a reduction of the carrier energy-loss rate. Therefore, a good understanding of the optical-phonon decay processes into acoustic phonons is a key element to address self-heating in optoelectronic devices and to devise phonon engineering strategies to lessen its impact on device performance [7]. Additionally, the decay of LO zone-center phonons can generate a high population of large-wave-vector phonons which may facilitate momentum conservation in nonradiative Auger processes. As already mentioned, these processes may severely limit the internal quantum efficiency of InGaN laser diodes at high drive currents [5].

A general theoretical overview of optical phonon anharmonic decay is given in Ref. [8] for a range of cubic and hexagonal semiconductors. The analysis is based on the Debye model for acoustic branches and assumes linearly dispersive optical branches. It is clear from this analysis that due to the large phonon gap in $\mathrm{GaN}$, the relaxation of optical modes proceeds through the Ridley channel (asymmetric decay into one optical and one acoustic mode) [9]. The prediction of phonon lifetimes for $\mathrm{c}-\mathrm{GaN}$ based on this model could not be compared with measurements because of the lack of experimental data. Phonon lifetimes and decay channels can be studied by means of temperature-dependent Raman-scattering experiments [10]. Raman-scattering studies of the decay of zone-center phonons have been carried out on several wurtzitetype semiconductors [11-13] and, more specifically, studies of wurtzite-type GaN have been extensively reported [14-18]. The analysis of the temperature dependence of the phonon linewidths is generally carried out by fitting a simplified theory of anharmonic decay in which, to the lowest order, the frequency shift and the broadening of the phonon line shapes 
is proportional to the sum of Bose-Einstein factors associated with the phonon decay products. In some cases $[14,16]$, the data is modeled using the simple Klemens channel (symmetric decay into acoustic phonons of the same energy) [19], which is clearly inconsistent with the large phonon gap in GaN.

In a previous work [13], we analyzed the anharmonic decay of the optical phonons in InN using the full Cowley's model [20], in which the anharmonic renormalization of the phonon frequencies and lifetimes is evaluated from the complex phonon self-energy. The phonon linewidth is given by the imaginary part of the self-energy, which is proportional to the two-phonon density of states (2PDOS), whereas the real part of the self-energy yields the anharmonic frequency shift. Therefore, linewidth and frequency shift are not separate magnitudes that can be fitted by independent parameters, but they are connected through the Kramers-Kronig relations.

In this paper, we present a Raman-scattering study of the temperature dependence of the optical phonons in c-GaN and we analyze their anharmonic decay on the basis of Cowley's anharmonic theory and the 2PDOS obtained from $a b$ initio calculations. The measurements were carried out over a wide temperature range up to $750 \mathrm{~K}$, and it was found that a significant contribution of higher-order decay processes had to be taken into account to explain the superlinear broadening of the LO mode with increasing temperature. The temperatureinduced downshift of the LO mode predicted by Cowley's theory is in excellent agreement with the observed temperature dependence of the Raman spectra. Similarly to the $E_{2}^{\text {high }}$ mode of the wurtzite phase [18], the transverse-optical (TO) mode of the c-GaN exhibits a weak temperature dependence and its frequency downshift is mainly governed by the lattice expansion.

\section{EXPERIMENT}

A $600-n m-t h i c k$ c-GaN layer with the zinc-blende structure was grown on a semi-insulating (001) GaAs substrate by plasma-assisted molecular-beam epitaxy (PA-MBE) in a MOD-GENII system using $\mathrm{As}_{2}$ as a surfactant to initiate the growth of the cubic phase material. To improve the quality of the c-GaN layer, a GaAs buffer layer was grown on the GaAs substrate. Zinc-blende GaN layers were grown at temperatures $\sim 680^{\circ} \mathrm{C}$ under Ga-rich PA-MBE conditions. The growth of the metastable polytype of GaN was initiated by epitaxy on cubic (001) GaAs substrates and by supplying an additional $\mathrm{As}_{2}$ flux [21].

The Raman-scattering experiments were carried out in backscattering configuration from a (001) face. The Raman spectra were excited with the $532 \mathrm{~nm}$ line of a diode-pumped Nd:YAG solid-state laser. The scattered light was analyzed using a Jobin-Yvon T64000 triple spectrometer equipped with a $\mathrm{LN}_{2}$-cooled charge-coupled device (CCD) detector. Raman spectra were obtained in the subtractive configuration of the spectrometer with $100 \mu \mathrm{m}$ slits, corresponding to a spectral slit width of $\sim 2.2 \mathrm{~cm}^{-1}$. The intrinsic phonon linewidths were obtained from the full width at half maximum (FWHM) of the Raman peaks using the Voigt profile approximation [13].

The sample temperature was varied between 80 and $390 \mathrm{~K}$ using a $\mathrm{LN}_{2}$ cryostat. In this temperature range, the Raman measurements were carried out in macrocamera configuration, with an incident power of $\sim 50 \mathrm{~mW}$. No heating effects were observed at this excitation power. A Linkam THMS600 high-temperature stage was employed to vary the sample temperature from $300 \mathrm{up}$ to $750 \mathrm{~K}$. In this temperature range, the measurements were performed using a confocal microscope with a long-working-distance $\times 80$ objective protected with a cooling jacket. In the micro Raman measurements, the incident power on the sample was kept below $5 \mathrm{~mW}$ to minimize heating effects. The sample temperature was monitored by a thermocouple attached to the sample holder block very close to the sample location. To ensure good thermal contact, the sample was attached to the sample holder block using an In droplet.

\section{RAMAN SPECTRA OF c-GaN}

Figure 1 shows the $z(x y) \bar{z}$ Raman spectra of $\mathrm{c}-\mathrm{GaN}$ recorded at three different temperatures. The spectra are dominated by the symmetry-allowed LO mode. The LO frequency decreases from $740 \mathrm{~cm}^{-1}$ at $120 \mathrm{~K}$ to $725 \mathrm{~cm}^{-1}$ at $670 \mathrm{~K}$. A weak shoulder can be seen on the low-energy

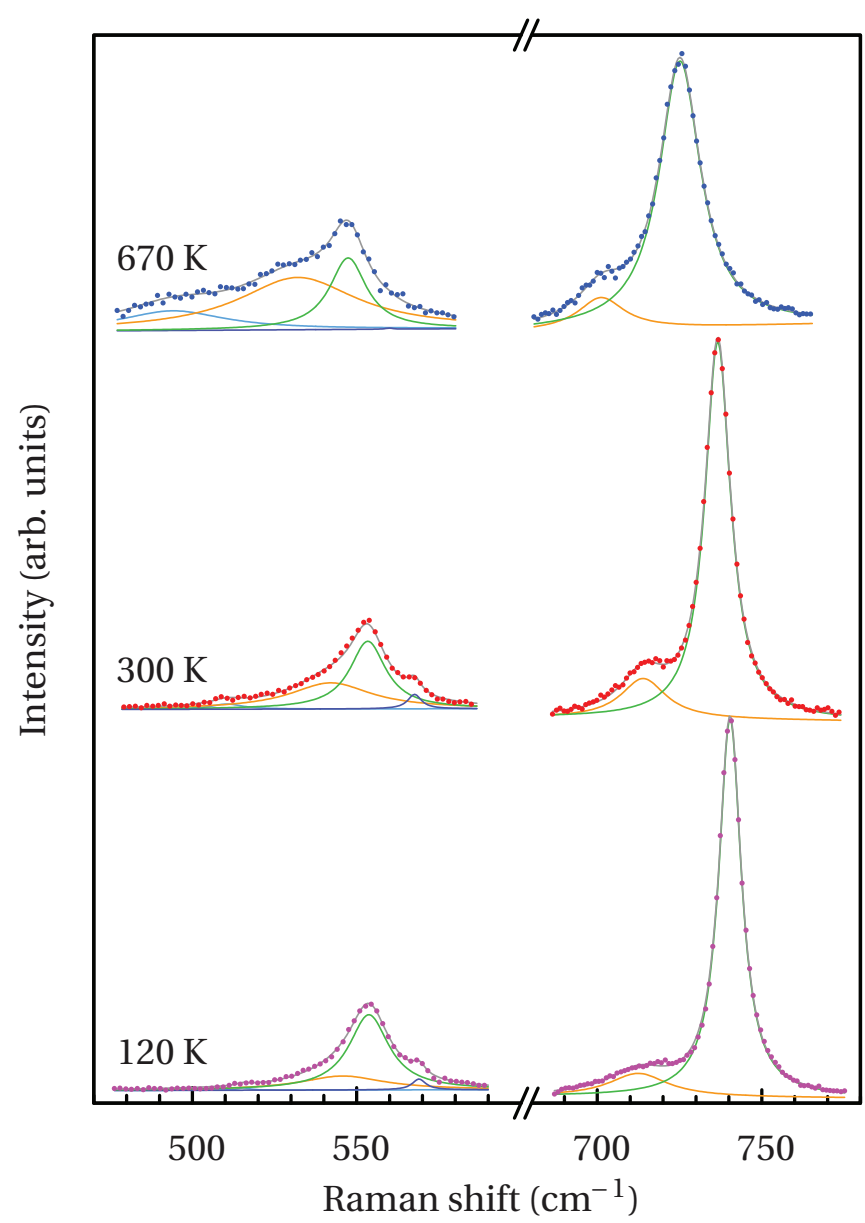

FIG. 1. (Color online) Raman spectra (filled circles) of the c$\mathrm{GaN}$ layer in the TO and LO frequency ranges obtained at three different temperatures. The solid lines are Lorentzian line-shape fits to separate the contributions of second-order bands (orange line) and the residual $E_{2}^{\text {high }}$ mode of wurtzite-GaN inclusions observed on the high-frequency side of the TO peak (dark blue line). 
side of this peak. To accurately determine the FWHM of the LO peak, Lorentzian line-shape fits of the Raman spectra were carried out, which are displayed in Fig. 1 as solid lines. Subsidiary modes in the low-energy side of the LO Raman peak were previously observed in the spectra of c-GaN and attributed to two-phonon combination bands and boundarylayer modes at the substrate interface [22]. According to our $a b$ initio calculations (see Sec. IV A), the frequency of the observed feature agrees well with a second-order $\mathrm{TA}(L)+\mathrm{TO}(L)$ combination mode. The intensity decrease of the low-energy mode relative to the LO mode as the temperature is lowered is well described by the phonon occupation ratio $\{1+n[\mathrm{TA}(L)]\}\{1+n[\mathrm{TO}(L)]\} /\{1+n[\mathrm{LO}(\Gamma)]\}$, where $n(\omega)$ is the Bose-Einstein occupation factor for the mode $\omega$, which supports the assignment of this feature to a second-order $\mathrm{TA}(L)+\mathrm{TO}(L)$ combination mode.

Although the TO mode is symmetry forbidden in backscattering from a (001) face, a weak Raman signal from the TO mode appears in the spectra. Since the c-GaN layer is transparent for the excitation laser beam, a measurable TO signal arises from the forward-scattering component induced by the laser beam that is reflected at the GaN/substrate interface [23]. As can be seen in Fig. 1, the Raman spectra around the TO phonon frequency of c-GaN contain the contributions of several Raman bands. Their relative contributions are illustrated by the Lorentzian line-shape fits displayed as continuous lines in Fig. 1. In addition to the dominant TO peak (green line), a weak narrow peak can be observed on its high-energy side. The frequency of this feature corresponds to the $E_{2}^{\text {high }}$ frequency of the hexagonal $\mathrm{GaN}$ and indicates the presence of a very small amount of hexagonal inclusions in the c-GaN layer. A broad band is also observed at about $12 \mathrm{~cm}^{-1}$ below the TO frequency (orange line). Since the relative intensity of this band decreases as the temperature is lowered, we attribute it to second-order scattering. Consistent with the observation of this band, our $a b$ initio calculations reveal a maximum of the $2 \mathrm{PDOS}$ at $\sim 15 \mathrm{~cm}^{-1}$ below the TO frequency that is associated with $\mathrm{TA}(X)+\mathrm{LA}(X)$ combination modes.

\section{RESULTS AND DISCUSSION}

\section{A. Lattice dynamics: $\boldsymbol{A b}$ initio calculations}

The accurate modeling of anharmonic decay processes requires a good knowledge of the lattice dynamics. In this section, we present $a b$ initio calculations based on perturbation density functional theory (PDFT) that give access to the phonon dispersion, phonon density of states (PDOS), and material parameters such as the bulk modulus and the mode Grüneisen parameter. These results will be key elements for the detailed analysis of the anharmonic phonon decay presented in Sec. IV B.

The PDFT calculations were carried out using a plane-wave pseudopotential approach in the local density approximation as implemented in the ABINIT code [24]. The $3 d$ electrons were included as valence states in the Ga pseudopotential, and reciprocal-space integrations were performed using a $6 \times 6 \times 6$ Monkhorst-Pack grid with an energy cutoff of $80 \mathrm{Ha}$. A full structural relaxation of the c-GaN lattice yields a lattice parameter of $a=4.499 \AA$, in good agreement with the value $a=4.501 \AA$ reported from x-ray measurements in bulk freestanding c-GaN [21]. The lattice parameter of c-GaN is substantially smaller than that of the GaAs substrate (5.65 $\AA$ ). However, due to the occurrence of a coincidencelattice mismatch $\left(p a_{\mathrm{GaAs}}-q a_{\mathrm{GaN}}\right) / p a_{\mathrm{GaAs}}$ which is negligible for $p=4, q=5$, the large misfit between lattice parameters can be accommodated by an array of pure edge dislocations with a period of $5 \mathrm{GaN}$ lattice planes, and thus the residual lattice misfit in the heterostructure is drastically reduced [25]. Therefore, in agreement with previous studies [26], we assume that there is no built-in strain in the $\mathrm{c}-\mathrm{GaN}$ layers.

To account for the lattice thermal-expansion effects on the phonon frequencies, we need the mode Grüneisen parameters of c-GaN, $\gamma=(B / \omega)(\partial \omega / \partial P)$, where $B=-V(\partial V / \partial P)^{-1}$ is the bulk modulus. From the DFT calculations, we obtain $B=$ 195.4 , and $\gamma_{\mathrm{LO}}=1.455$ and $\gamma_{\mathrm{TO}}=1.222$. These values are in excellent agreement with the experimental values reported by Goñi et al. [26].

For the calculation of phonon frequencies, the dynamical matrix was obtained on a mesh of $k$ points using the perturbation-theory linear-response approach [27], which allows the interpolation to arbitrary wave vectors for the calculation of phonon dispersion and PDOS. Figure 2 shows the phonon dispersion of $\mathrm{c}-\mathrm{GaN}$ along the principal symmetry directions and the corresponding PDOS. Due to the large difference in the masses of $\mathrm{Ga}$ and $\mathrm{N}$ atoms, there is a large gap of $\sim 200 \mathrm{~cm}^{-1}$ between the optical branches, which pertain to predominantly nitrogenlike vibrations, and the acoustical branches, which consist of mainly galliumlike vibrations. The PDOS exhibits maxima that correspond to van Hove singularities in the phonon dispersion. These are labeled in the PDOS plot displayed in Fig. 2 and will be used to identify the most likely decay channels for the optical modes of $\mathrm{c}-\mathrm{GaN}$. It should be noted that because of the limitations of the local-density approximation (LDA) calculations (underestimation of the band gap and overestimation of the dielectric constant), the calculated LO frequency $\left(719.1 \mathrm{~cm}^{-1}\right)$ is $22 \mathrm{~cm}^{-1}$ lower than the experimental value measured at $80 \mathrm{~K}$. This discrepancy stems from the overestimation of the dielectric constant by the DFT calculations, which yield a value of $\varepsilon_{\infty}=6.078$, noticeably higher than the reported experimental value $\varepsilon_{\infty}^{\text {expt }}=5.31$ [3]. Since the LO-TO splitting is given by

$$
\omega_{\mathrm{LO}}^{2}-\omega_{\mathrm{TO}}^{2}=\frac{4 \pi Z^{* 2}}{V_{0} \mu \varepsilon_{\infty}},
$$

where $Z^{*}$ is the effective Born charge, $V_{0}$ is the unit cell volume, and $\mu$ is the reduced mass, the overestimation of $\varepsilon_{\infty}$ gives rise to an underestimation of the LO-TO splitting. We can account for this effect by evaluating Eq. (1) using $\varepsilon_{\infty}^{\text {expt }}$. Then we obtain $\omega_{\mathrm{LO}}=740.8 \mathrm{~cm}^{-1}$, which is quite close to the LO frequency we measured at $80 \mathrm{~K}$. Accordingly, the analysis of the anharmonic decay channels and the corresponding shift and broadening of the Raman peaks will be performed consistently using the results of the DFT calculations, bearing in mind that the comparison with the experimental frequencies will be effected by means of a rigid shift of the temperature-dependent frequency curves. The phonon frequencies obtained from 

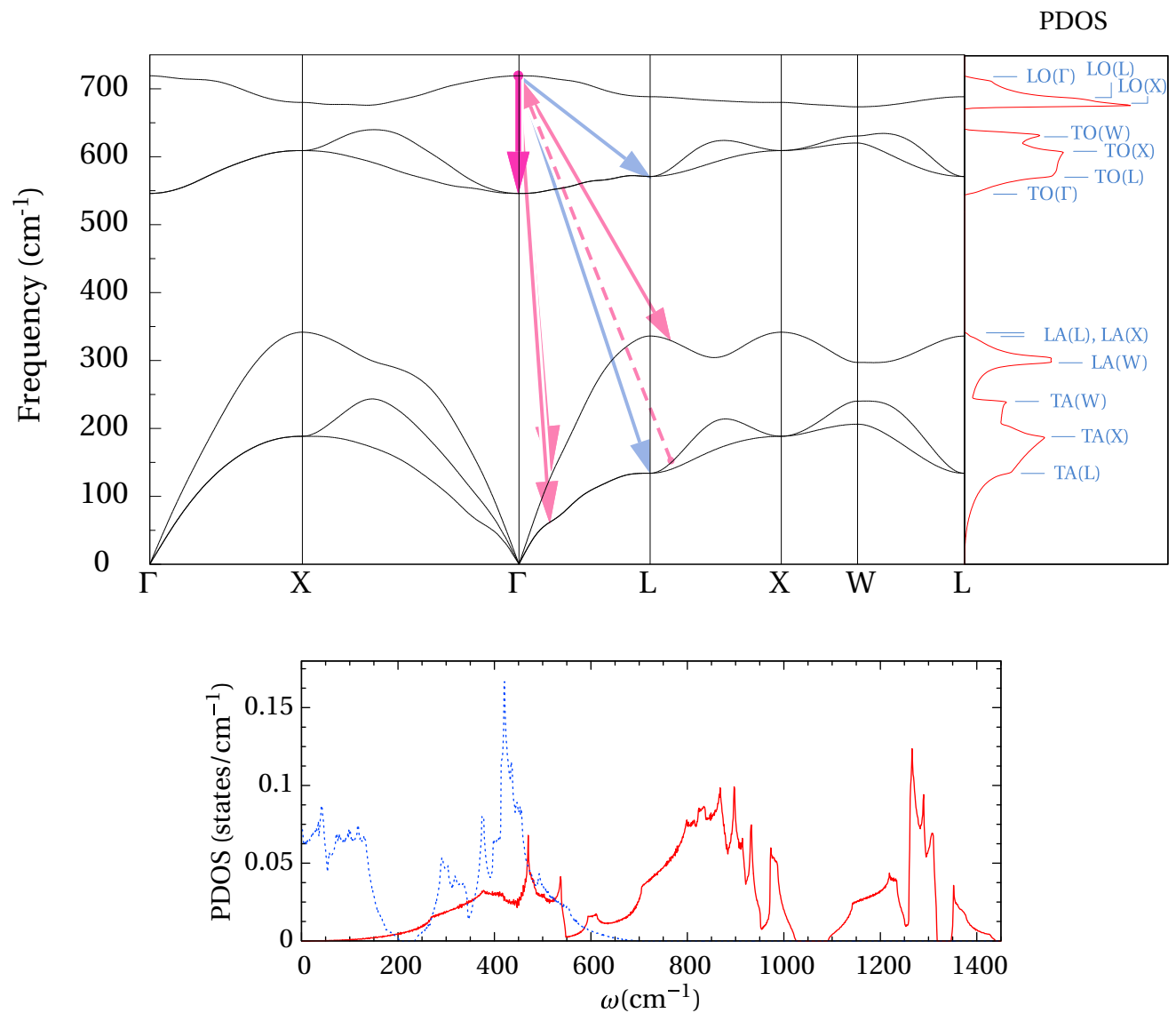

FIG. 2. (Color online) Upper panel: Phonon dispersion and phonon density of states in c-GaN as obtained from DFT calculations. The arrows indicate the main decay channels for the zone-center LO mode discussed in the text. Blue arrows indicate the three-phonon decay process considered and magenta arrows indicate two possible four-phonon decay processes. Lower panel: Two-phonon sum (solid line) and difference (dotted line) density of states.

DFT calculations at the principal high-symmetry points are summarized in Table I.

In Fig. 2 (lower panel), we display the 2PDOS corresponding to the sum and phonon differences that will be used in Sec. IV B to evaluate the effects of decay into combinations of two zone-edge phonons in Cowley's anharmonicity model. The 2PDOS for sums is about four times higher around the LO frequency than around the TO frequency, whereas the 2PDOS for differences is vanishing around the LO frequency. The 2PDOS for sums exhibits steep slopes at the TO and LO frequencies which, as we discuss in Sec. IV B, introduce a

TABLE I. Phonon frequencies at the principal high-symmetry points as obtained by DFT calculations.

\begin{tabular}{lccrr}
\hline \hline & \multicolumn{4}{c}{ Phonon frequency $\left(\mathrm{cm}^{-1}\right)$} \\
\cline { 2 - 5 } Symmetry point & TO & LO & \multicolumn{1}{c}{ TA } & LA \\
\hline$\Gamma$ & 545.9 & 719.1 & 0.0 & 0.0 \\
$X$ & 609.0 & 679.9 & 188.3 & 341.4 \\
$L$ & 570.8 & 688.3 & 133.9 & 335.9 \\
$W$ & 620.1 & 673.4 & 206.2 & 297.1 \\
& 630.5 & & 240.1 & \\
\hline \hline
\end{tabular}

frequency dependence in the anharmonicity corrections that must be taken into account in the line-shape modeling.

\section{B. Theoretical model for anharmonic decay}

The anharmonic coupling between the eigenstates of the harmonic lattice Hamiltonian (bare phonons) leading to a finite lifetime and frequency renormalization of the phonons was described in the perturbation-theory framework by Cowley [20]. Cowley's theory was successfully applied to analyze phonon decay in several temperature-dependent Raman scattering studies of $\mathrm{Si}, \mathrm{Ge}, \alpha$-Sn [10], and InN [13], and has revealed subtle effects in the temperature behavior of Raman scattering of compound semiconductors such as AlAs [28] and $\mathrm{ZnO}[12,29]$.

The anharmonic theory has been discussed at length in the literature [10,20,30]. We present here just an overview of the most relevant points that will be used later in the analysis of our data. To the lowest order in perturbation theory, the imaginary part of the phonon self-energy can be written as

$$
\begin{aligned}
\Gamma(\omega)= & \left|V_{3}^{+}\right|^{2}\left[1+n\left(\omega_{1}^{+}\right)+n\left(\omega_{2}^{+}\right)\right] \rho^{+}(\omega) \\
& +\left|V_{3}^{-}\right|^{2}\left[n\left(\omega_{2}^{-}\right)-n\left(\omega_{1}^{-}\right)\right] \rho^{-}(\omega),
\end{aligned}
$$


where $V_{3}^{+}$and $V_{3}^{-}$are, respectively, the effective cubic anharmonic potentials for decay into a phonon $\operatorname{sum}\left(\omega \rightarrow \omega_{1}^{+}+\omega_{2}^{+}\right)$ and difference $\left(\omega \rightarrow \omega_{2}^{-}-\omega_{1}^{-}\right)$, and $\rho^{+}(\omega)$ and $\rho^{-}(\omega)$ are the two-phonon DOS for phonon sums and differences, respectively. Energy and wave vector must be conserved in the phonon decay process. The effective cubic anharmonic potentials are related to the third-order derivatives of the total energy and to the harmonic frequencies and eigenvectors. All of these quantities can be evaluated using PDFT by computing the linear response to atomic displacements from the equilibrium position. Indeed, full $a b$ initio calculations of phonon linewidths were reported on III-V semiconductors [31]. These PDFT calculations deal only with the third-order derivatives of the total energy as obtained from the $2 n+1$ theorem (lowest-order anharmonicity correction). Furthermore, for the LO modes, the contribution to the anharmonic dynamical tensor due to the electron density response to an electric field and the corresponding nonanalytic wave-function response are neglected. While a good agreement with experimental data is generally found at low temperatures, significant deviations may occur at higher temperatures because of the increasing importance of higher-order corrections. In view of the computational complexity of the full ab initio approach and its intrinsic limitations, to analyze our temperature-dependent data we have adopted a semiempirical approach similar to that used by Serrano et al. [29], in which the effective anharmonic potentials are considered as adjustable parameters while PDFT is employed to obtain the phonon frequencies and density of states.

Higher-order terms (quartic anharmonicity) involving fourphonon processes were analyzed by Balkanski et al. [30] and found to be relevant to explain the superlinear temperature dependence of the optical phonon broadening in $\mathrm{Si}$, particularly at higher temperatures. Although temperature-dependence fits based on the occupation factors indicate the importance of higher-order terms in many cases $[11,15,16,18]$, the general expressions for the higher-order terms are, however, difficult to evaluate and therefore it is not trivial to extend Cowley's theory to higher order. In our previous anharmonicity study on InN [13], we restricted ourselves to the higher-order decay channels involving the decay into a zone-center phonon $\left(\omega_{1}\right)$ and a pair of opposite wave-vector phonons at zone edge $\left(\omega_{2}\right.$, $\left.\omega_{3}\right)$ in an attempt to take into account higher anharmonic terms in Cowley's formalism. Under these constraints, the quartic anharmonicity contribution reduces to [13]

$$
\begin{aligned}
\Gamma^{(4)}(\omega) \propto & \left|\tilde{V}_{4}^{+}\right|^{2}\left\{\left[n\left(\omega_{1}^{+}\right)+1\right]\left[n\left(\omega_{2}^{+}\right)+1\right]\left[n\left(\omega_{3}^{+}\right)+1\right]\right. \\
& \left.-n\left(\omega_{1}^{+}\right) n\left(\omega_{2}^{+}\right) n\left(\omega_{3}^{+}\right)\right\} \rho^{+}\left(\omega-\omega_{1}^{+}\right) \\
& +\left|\tilde{V}_{4}^{-}\right|^{2}\left\{\left[n\left(\omega_{1}^{-}\right)+1\right]\left[n\left(\omega_{2}^{-}\right)+1\right] n\left(\omega_{3}^{-}\right)\right. \\
& \left.-n\left(\omega_{1}^{-}\right) n\left(\omega_{2}^{-}\right)\left[n\left(\omega_{3}^{-}\right)+1\right]\right\} \rho^{-}\left(\omega-\omega_{1}^{-}\right),
\end{aligned}
$$

where $\tilde{V}_{4}^{+}, \tilde{V}_{4}^{-}$are effective anharmonic potentials to be considered as empirical adjustable parameters. Once the 2 PDOS is known and the main decay channels have been identified, Eqs. (2) and (3) allow us to evaluate the phonon linewidth broadening (imaginary part of the phonon selfenergy). Since the real and imaginary parts of the phonon self-energy are related by the Kramers-Kronig relations, the anharmonic frequency shift is obtained as

$$
\Delta(\omega)=-\frac{2}{\pi} \mathcal{P} \int_{0}^{\infty} \frac{\omega^{\prime}}{\omega^{\prime 2}-\omega^{2}} \Gamma\left(\omega^{\prime}\right) d \omega^{\prime} .
$$

In addition to the anharmonic shift, the actual temperature dependence of the phonon frequency also contains an important contribution from the thermal expansion of the lattice, which can be estimated from the linear thermal-expansion coefficient $\alpha(T)$ and the mode Grüneisen parameter $\gamma$ as

$$
\omega(T)=\omega(80 \mathrm{~K})-3 \omega_{0} \gamma \int_{80}^{T} \alpha\left(T^{\prime}\right) d T^{\prime} .
$$

For the linear thermal expansion of $\mathrm{c}-\mathrm{GaN}$, we consider $\alpha \approx$ $\frac{1}{3}\left(2 \alpha_{a}+\alpha_{c}\right)$, where $\alpha_{a}$ and $\alpha_{c}$ are the linear thermal-expansion coefficients of hexagonal GaN reported by Roder et al. [32]. Given the similar thermal expansion of $\mathrm{GaN}$ and GaAs (the respective thermal expansion coefficients differ by less than $\sim 20 \%$ at the growth temperature) [32,33] and the stress relaxation at the $\mathrm{GaN} / \mathrm{GaAs}$ interface, we neglect the effects of differential thermal expansion, although a small residual compressive strain might be present in the c-GaN layer.

To obtain the phonon frequency and FWHM curves vs $T$, the Raman line shape is calculated taking into account the self-energy corrections $[10,13]$, and the peak frequency and FWHM are determined for each temperature. The temperature dependence of the phonon linewidth can then be fitted to the Raman data to obtain the anharmonic potentials $V_{3}^{+}, V_{3}^{-}, \tilde{V}_{4}^{+}$, and $\tilde{V}_{4}^{-}$. These potentials determine not only the broadening of the phonon line, but also the anharmonic frequency shift which is given by the real part of the phonon self-energy.

\section{Phonon decay of the LO mode}

By inspection of the PDOS (Fig. 2) and the phonon frequencies at the high-symmetry points (Table I), we conclude that the only three-phonon process that verifies energy conservation is the Ridley channel $\mathrm{LO}(\Gamma) \rightarrow \mathrm{TO}(L)+\mathrm{TA}(L)$. This channel is illustrated by the blue arrows in Fig. 2. Detailed measurements of the LO-mode linewidth were performed at temperature intervals of about $20 \mathrm{~K}$ and the intrinsic FWHM of the LO phonon is plotted in Fig. 3(a) as a function of temperature. Error bars have been set according to the frequency separation between consecutive pixels in the CCD detector. The experimental data show a superlinear increase of the FWHM as temperature is increased which cannot be accounted for solely by the above asymmetric Ridley channel. In this case, the lower-energy phonon decay product $[\mathrm{TA}(L)]$ has an energy lower than $k_{B} T$ for most of the temperature range and therefore its occupation factor dominates and yields a nearly linear temperature dependence. This behavior is illustrated by the dashed line in Fig. 3(a), which depicts the temperature dependence of the FWHM taking into account only the Ridley channel and the background contribution $\Gamma_{0}$. Therefore, further channels have to be considered to explain the observed FWHM behavior. In the spirit of Eq. (3), we consider fourphonon processes involving the decay into the zone-center TO phonon and sums or differences of acoustic phonons. Two such processes verifying energy conservation can be readily identified by inspection of the phonon dispersion, and they are depicted by magenta arrows in Fig. 2. First, a process leading 

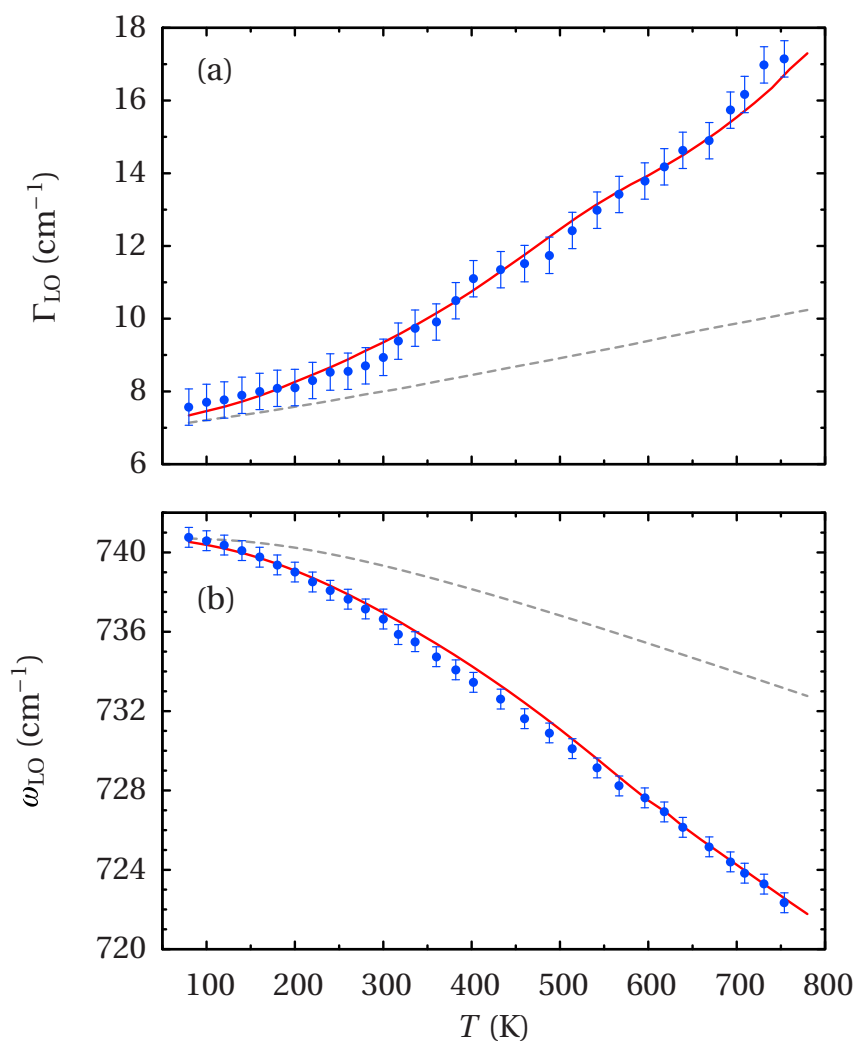

FIG. 3. (Color online) (a) Temperature dependence of the LO phonon linewidth (filled circles). The solid line is a fit of the anharmonicity model including three- and four-phonon processes. The dashed line has been obtained by considering only the threephonon processes. (b) Temperature dependence of the LO phonon frequency (filled circles). The frequency shift due to the thermal lattice expansion is indicated by the dashed line. The calculated temperature dependence taking into account both the thermal expansion and the anharmonic renormalization of the phonon frequency is plotted as a solid line.

to the asymmetric creation of acoustic modes, $\mathrm{LO}(\Gamma) \rightarrow$ $\mathrm{TO}(\Gamma)+\mathrm{LA}(\Gamma-L)+\mathrm{TA}(\Gamma-L)$, where the frequencies of the TA and LA modes are $\sim 58$ and $\sim 116 \mathrm{~cm}^{-1}$, respectively. An analogous decay channel involving the TA and LA acoustic branches for points along the $\Gamma-X$ line also exists. Second, a process involving up-conversion of TA modes (indicated by the dashed arrow in Fig. 2), $\operatorname{LO}(\Gamma) \rightarrow \mathrm{TO}(\Gamma)+\operatorname{LA}(\hat{L})-\mathrm{TA}(\hat{L})$, where $\hat{L}$ denotes points close to the $L$ point along the $L-X$ (or $L-W$ ) lines that verify energy conservation. Taking into account these decay channels, Eqs. (2) and (3) of the anharmonicity model have been fitted to the experimental data using the anharmonic potentials as fitting parameters. An additive contribution $\Gamma_{0}$ has been included to account for scattering with defects and impurities. A good fit to the data, plotted as a solid line in Fig. 3(a), is obtained for $\left|V_{3}^{+}\right|^{2}=10 \mathrm{~cm}^{-2}$, $\left|\tilde{V}_{4}{ }^{+}\right|^{2}=17 \mathrm{~cm}^{-2},\left|\tilde{V}_{4}^{-}\right|^{2}=22 \mathrm{~cm}^{-2}$, and $\Gamma_{0}=6.3 \mathrm{~cm}^{-1}$. The background contribution $\Gamma_{0}$ is significantly larger than the values typically obtained for InN (Ref. [13]) or wurtzite-GaN (Refs. [15,18]). Depending on the sample quality and the range of temperatures fitted, widely varying results for the $\Gamma_{0}$ value were found for the $A_{1}$ (LO) mode of wurtzite-GaN.
A study of a $2.2-\mu \mathrm{m}$-thick metal-organic vapor-phase epitaxy sample in the temperature range of 85 to $760 \mathrm{~K}$ yielded a background linewidth contribution of $\Gamma_{0}=2.3 \mathrm{~cm}^{-1}[15]$, whereas later studies performed on high-quality hydride vapor epitaxy layers with thickness $\sim 80 \mu \mathrm{m}$ in the temperature range of 20 to $235 \mathrm{~K}$ found $\Gamma_{0}=0.9 \mathrm{~cm}^{-1}$ [18]. Much higher values for the background linewidth contribution $\left(\Gamma_{0}=5.7 \mathrm{~cm}^{-1}\right)$ were reported for AIN [15]. The growth of the zinc-blende metastable polytype of $\mathrm{GaN}$ is challenging and the resulting material usually contains a significant density of stacking faults and wurtzite-type inclusions. The presence of a higher density of defects in the c-GaN layers limits the phonon coherence length and results in substantially broadened phonon line shapes. Such a broadening depends on the sample quality and noticeable variations were found in preliminary measurements performed on several c-GaN samples obtained in different MBE runs. The sample with the smallest $A_{1}$ (LO) FWHM was selected to perform the temperature-dependent measurements presented in this work.

As can be seen in Fig. 3(b), the LO phonon exhibits a frequency downshift of $19 \mathrm{~cm}^{-1}$ when the temperature is increased from 80 to $780 \mathrm{~K}$. More than one-third of the downshift is accounted for by the thermal expansion of the lattice. The thermal-expansion contribution to the LO frequency downshift as calculated from Eq. (5) is plotted with a dashed line. The solid line is obtained by adding the anharmonic renormalization of the phonon self-energy [Eq. (4)] calculated with the same set of parameters determined from the FWHM fit. The theoretical curve for the temperature dependence of the LO frequency closely matches the experimental points. This confirms our assumption that thermally induced built-in strains are negligible in these c-GaN layers.

\section{Phonon decay of the TO mode}

As already discussed in Sec. III, a sizable signal from the TO mode is also observed in the backscattering measurements on the (001)-oriented c-GaN layers. However, since it overlaps second-order bands and the residual $E_{2}^{\text {high }}$ signal from wurtzite inclusions, an accurate determination of the TO line-shape parameters is difficult. As shown in Fig. 1, a four-Lorentzian line-shape fit was performed in the TO spectral region to extract the peak frequency and the FWHM of the TO Raman peak. The results are plotted in Fig. 4, where larger error bars have been included to account for the uncertainties introduced by the fitting procedure. The FWHM data shows some scatter around $7 \mathrm{~cm}^{-1}$, but there is no clear trend with increasing temperature, which suggests that anharmonic renormalization effects are notably weak for this mode. This is confirmed by the relatively small downshift of the TO frequency over the temperature range studied, which, as can be seen in Fig. 4(b), can be entirely accounted for by the thermal-expansion contribution (dashed line).

According to the phonon dispersion of c-GaN (see Fig. 2), the most likely channel for TO phonon decay is the asymmetric decay into two acoustic phonons: $\mathrm{TO}(\Gamma) \rightarrow \mathrm{LA}(W)+$ $\mathrm{TA}^{+}(W)$, where $\mathrm{TA}^{+}(W)$ stands for the upper transverse acoustic branch at $W$. A fit to the FWHM Raman data, displayed as a solid line in Fig. 4(a), yields $\left|V_{3}^{+}\right|^{2}=6 \mathrm{~cm}^{-2}$ and $\Gamma_{0}=6.5 \mathrm{~cm}^{-1}$. The background contribution $\Gamma_{0}$ agrees 

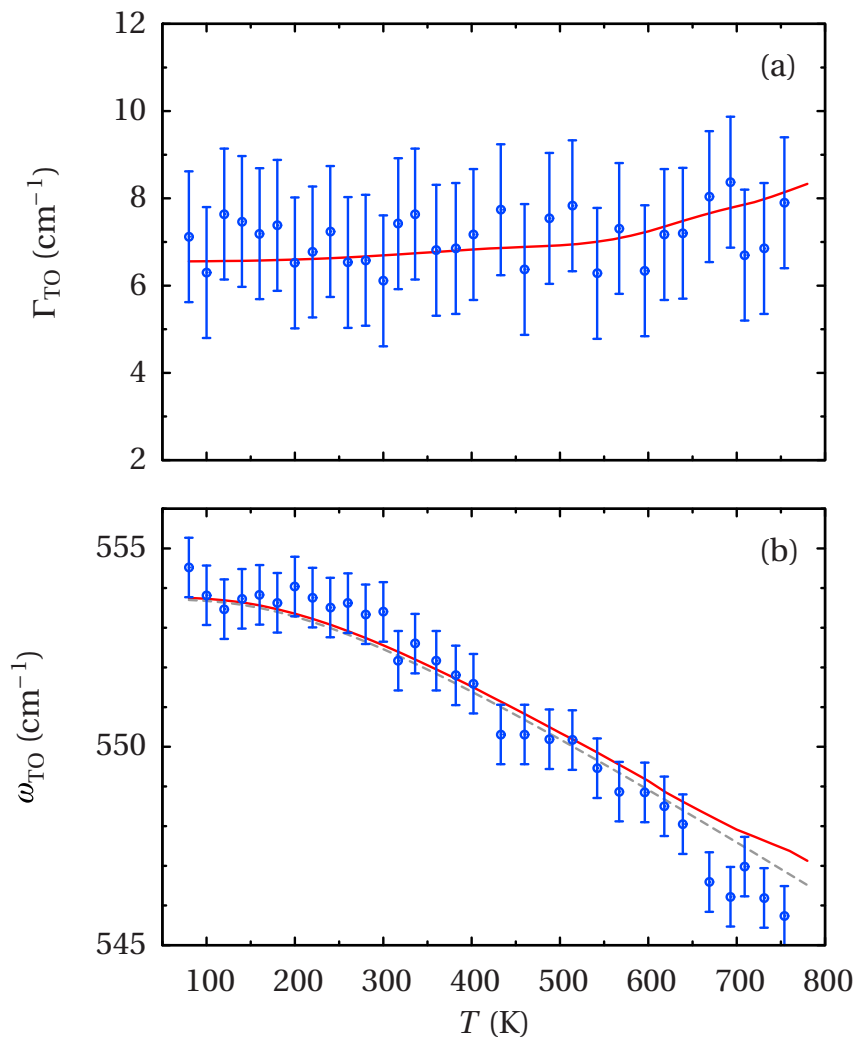

FIG. 4. (Color online) (a) Temperature dependence of the TO phonon linewidth (filled circles). The solid line is a fit of the anharmonicity model taking into account the asymmetric decay channel into acoustic phonons of the LA and upper TA branches around $W$. (b) Temperature dependence of the TO phonon frequency (filled circles). The frequency shift predicted by the model calculations (solid line) is basically accounted for by the thermal lattice expansion, indicated by the dashed line.

well with the value obtained for the LO model. A very weak temperature dependence was also reported for the $E_{2}^{\text {high }}$ mode in wurtzite $\mathrm{GaN}$ [18], which corresponds to the zone-folded $\mathrm{TO}(L)$ mode of the zinc-blende phase, and it was attributed to a higher-order decay mechanism. In contrast, a three-phonon decay channel exists for the TO mode of $\mathrm{c}-\mathrm{GaN}$. Then, the weak temperature dependence of the $\mathrm{TO}$ mode of $\mathrm{c}-\mathrm{GaN}$ is explained instead by the combined effect of a relatively weak anharmonic coupling potential, the fact that the 2PDOS is very low around the TO frequency [as can be seen in Fig. 2(b)], and the unavailability of relevant higher-order decay channels.

\section{E. LO phonon lifetime}

In polar semiconductors, the LO phonon lifetime is a key parameter for carrier-lattice thermalization, as the excess energy of the carriers is removed by Fröhlic interaction with LO phonons. The phonon lifetime can be studied in the frequency domain by means of Raman spectroscopy. The phonon lifetime is related to the Raman linewidth through the energy-time uncertainty relation $\tau^{-1}=\Gamma / \hbar$, where $\hbar=$ $5.309 \times 10^{-12} \mathrm{~cm}^{-1} \mathrm{~s}$ is Plank's constant. The finite phonon linewidth measured in Raman experiments is determined both by the anharmonic decay into other phonons with a charac-

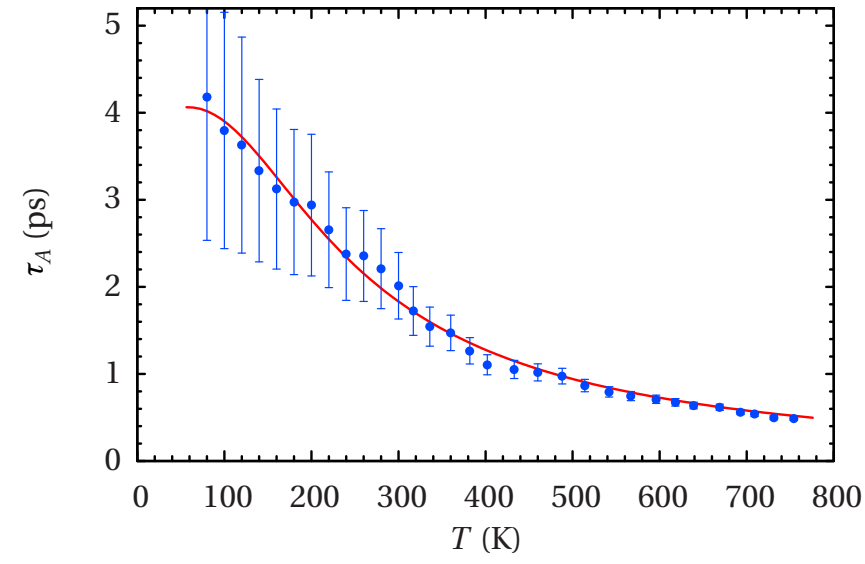

FIG. 5. (Color online) LO phonon lifetimes at $80 \mathrm{~K}$ and room temperature as determined from the linewidth analysis of the Raman spectra. The solid line is a guide to the eye obtained from the occupation factors in Eqs. (2) and (3).

teristic time $\tau_{A}$ and by the perturbation of the translational symmetry of the lattice due to defects and impurities. The latter yields a temperature-independent background contribution $\Gamma_{0}$ to the Raman FWHM that was determined in the model fits to the temperature-dependent FWHM data. If we associate a characteristic time $\tau_{B}=\hbar / \Gamma_{0}$ to this broadening mechanism, we may separate the anharmonic and background contributions to the phonon lifetime as

$$
\frac{1}{\tau}=\frac{1}{\tau_{A}}+\frac{1}{\tau_{B}} .
$$

The relaxation time $\tau_{A}$ describes the intrinsic phonon-lifetime limiting processes arising from anharmonic phonon decay and is therefore characteristic of the material and determined by its lattice dynamics. In contrast, the relaxation time $\tau_{B}$ describes phonon dephasing due to interactions with lattice defects and impurities, and therefore it depends on the crystalline quality of the sample. Given that a substantial fraction of the LO phonon linewidth is due to lattice disorder effects, the LO phonon dephasing time $\tau$ is expected to increase as growth methods are improved and higher-quality c-GaN crystals are produced. In Fig. 5, we plot the anharmonic decay time $\tau_{A}$ as a function of temperature as derived from the Raman FWHM using Eq. (6). The anharmonic decay time shows a sizable increase at lower temperatures, where a substantial fraction of the Raman linewidth is given by the background term $\Gamma_{0}$. Given the small value of the anharmonic component of the FWHM, the error bars on $\tau_{A}$ are increasingly large at lower temperatures. However, the central values show a regular trend that closely follows the solid line calculated by using the occupation factors appearing in Eqs. (2) and (3). This fact leads us to think that the anharmonic lifetime values determined from the Raman measurements are fairly accurate even at lower temperatures. We obtain 4.2 and 2.0 ps at low temperature and room temperature, respectively. These values are in excellent agreement with the values theoretically predicted by Barman and Srivastava [8]. The room-temperature LO phonon lifetime we find for $\mathrm{c}-\mathrm{GaN}$ is higher than the values around $0.9 \mathrm{ps}$ reported for the $A_{1}$ (LO) mode of wurtzite GaN [18,34]. The values of the anharmonic lifetimes are 
relevant for the buildup of a nonthermal phonon population and therefore for determining the hot-carrier relaxation dynamics by electron-phonon interaction. Given the relatively high values of anharmonic phonon lifetimes in $\mathrm{c}-\mathrm{GaN}$, hot-phonon effects may be expected to be significant in this material.

\section{SUMMARY AND CONCLUSIONS}

In this work, we have carried out Raman-scattering measurements on zinc-blende $\mathrm{GaN}$ over a temperature range from 80 to $750 \mathrm{~K}$. Anharmonic phonon decay modeling of the Raman line shapes has been performed by evaluating the complex phonon self-energy, taking into account the phonon dispersion and phonon density of states obtained from DFT calculations. The most likely decay channels have been identified from the phonon dispersion and PDOS obtained from the calculations.

The LO phonon FWHM displays a superlinear temperature dependence that can only be explained by considering higher-order processes in addition to the Ridley channel $\mathrm{LO} \rightarrow \mathrm{TO}(L)+\mathrm{TA}(L)$. With the aid of the calculated phonon dispersion, several four-phonon decay channels have been identified. The contribution of these higher-order processes is found to be more important than for the case of the wurtzite GaN. The LO frequency downshift with temperature is well accounted for by the combined effect of the thermal lattice expansion and the anharmonic renormalization of the phonon frequency. The TO mode, for which a three-phonon decay channel $\mathrm{TO}(\Gamma) \rightarrow \mathrm{LA}(W)+\mathrm{TA}^{+}(W)$ exists, displays a weak temperature dependence and its frequency downshift is mainly determined by the thermal lattice expansion effect. This implies a small anharmonic coupling for this mode.

The LO phonon lifetime has been evaluated from the temperature-dependent FWHM data, and it has been found to be in excellent agreement with previous theoretical estimations. The characteristic phonon decay rate in the $\mathrm{c}-\mathrm{GaN}$ layers studied contains a substantial background contribution arising from scattering by lattice defects which is significantly higher than for the wurtzite GaN. However, the anharmonic phonon decay rate is lower than in wurtzite GaN.

\section{ACKNOWLEDGMENT}

N.D. acknowledges financial support from the FPU program of the Spanish Ministerio de Educacion, Cultura y Deporte.
[1] S. Li, J. Schörmann, D. J. As, and K. Lischka, Appl. Phys. Lett. 90, 071903 (2007).

[2] C. J. M. Stark, T. Detchprohm, S. C. Lee, Y.-B. Jiang, S. R. J. Brueck, and C. Wetzel, Appl. Phys. Lett. 103, 232107 (2013).

[3] M. Feneberg, M. Röppischer, C. Cobet, N. Esser, J. Schörmann, T. Schupp, D. J. As, F. Hörich, J. Bläsing, A. Krost et al., Phys. Rev. B 85, 155207 (2012).

[4] D. J. As, D. Schikora, A. Greiner, M. Lübbers, J. Mimkes, and K. Lischka, Phys. Rev. B 54, R11118 (1996).

[5] K. T. Delaney, P. Rinke, and C. G. Van de Walle, Appl. Phys. Lett. 94, 191109 (2009).

[6] S. Hess, R. Taylor, E. O’Sullivan, J. Ryan, N. Cain, V. Roberts, and J. Roberts, Phys. Status Solidi B 216, 51 (1999).

[7] A. A. Balandin and D. L. Nika, Mater. Today 15, 266 (2012).

[8] S. Barman and G. P. Srivastava, Phys. Rev. B 69, 235208 (2004).

[9] B. K. Ridley, J. Phys. Condens. Matter 8, L511 (1996).

[10] J. Menéndez and M. Cardona, Phys. Rev. B 29, 2051 (1984).

[11] J. W. Pomeroy, M. Kuball, H. Lu, W. J. Schaff, X. Wang, and A. Yoshikawa, Appl. Phys. Lett. 86, 223501 (2005).

[12] R. Cuscó, E. Alarcón-Lladó, J. Ibáñez, L. Artús, J. Jiménez, B. Wang, and M. J. Callahan, Phys. Rev. B 75, 165202 (2007).

[13] N. Domènech-Amador, R. Cuscó, L. Artús, T. Yamaguchi, and Y. Nanishi, Phys. Rev. B 83, 245203 (2011).

[14] M. S. Liu, L. A. Bursill, S. Prawer, K. W. Nugent, Y. Z. Tong, and G. Y. Zhang, Appl. Phys. Lett. 74, 3125 (1999).

[15] A. Link, K. Bitzer, W. Limmer, R. Sauer, C. Kirchner, V. Schwegler, M. Kamp, D. G. Ebling, and K. W. Benz, J. Appl. Phys. 86, 6256 (1999).

[16] W. S. Li, Z. X. Shen, Z. C. Feng, and S. J. Chua, J. Appl. Phys. 87, 3332 (2000).

[17] X.-B. Chen, J. Huso, J. L. Morrison, L. Bergman, and A. P. Purdy, J. Appl. Phys. 98, 026106 (2005).

[18] D. Y. Song, S. A. Nikishin, M. Holtz, V. Soukhoveev, A. Usikov, and V. Dmitriev, J. Appl. Phys. 101, 053535 (2007).
[19] P. G. Klemens, Phys. Rev. 148, 845 (1966).

[20] R. A. Cowley, Rep. Prog. Phys 31, 123 (1968).

[21] S. V. Novikov, N. Zainal, A. V. Akimov, C. R. Staddon, A. J. Kent, and C. T. Foxon, J. Vac. Sci. Technol. B 28, C3B1 (2010).

[22] G. Mirjalili, T. J. Parker, S. F. Shayesteh, M. M. Bülbül, S. R. P. Smith, T. S. Cheng, and C. T. Foxon, Phys. Rev. B 57, 4656 (1998).

[23] H. Siegle, L. Eckey, A. Hoffmann, C. Thomsen, B. Meyer, D. Schikora, M. Hankeln, and K. Lischka, Solid State Comm. 96, 943 (1995).

[24] X. Gonze, J.-M. Beuken, R. Caracas, F. Detraux, M. Fuchs, G.-M. Rignanese, L. Sindic, M. Verstraete, G. Zerah, F. Jollet et al., computer code ABINIT (Common project: Université Catholique de Louvain, Corning Inc., and other contributors), http://www.abinit.org; Comput. Mater. Sci. 25, 478 (2002).

[25] M. A. Herman, W. Richter, and H. Sitter, Epitaxy: Physical Principles and Technical Implementation, Springer Series on Materials Science (Springer-Verlag, Berlin-Heidelberg, 2004).

[26] A. R. Goñi, H. Siegle, K. Syassen, C. Thomsen, and J.-M. Wagner, Phys. Rev. B 64, 035205 (2001).

[27] X. Gonze and C. Lee, Phys. Rev. B 55, 10355 (1997).

[28] M. Canonico, C. Poweleit, J. Menéndez, A. Debernardi, S. R. Johnson, and Y.-H. Zhang, Phys. Rev. Lett. 88, 215502 (2002).

[29] J. Serrano, F. J. Manjón, A. H. Romero, F. Widulle, R. Lauck, and M. Cardona, Phys. Rev. Lett. 90, 055510 (2003).

[30] M. Balkanski, R. F. Wallis, and E. Haro, Phys. Rev. B 28, 1928 (1983).

[31] A. Debernardi, Phys. Rev. B 57, 12847 (1998).

[32] C. Roder, S. Einfeldt, S. Figge, and D. Hommel, Phys. Rev. B 72, 085218 (2005).

[33] J. S. Blakemore, J. Appl. Phys. 53, R123 (1982).

[34] S. Tripathy, S. J. Chua, and A. Ramam, J. Phys.: Condens. Matter 14, 4461 (2002). 